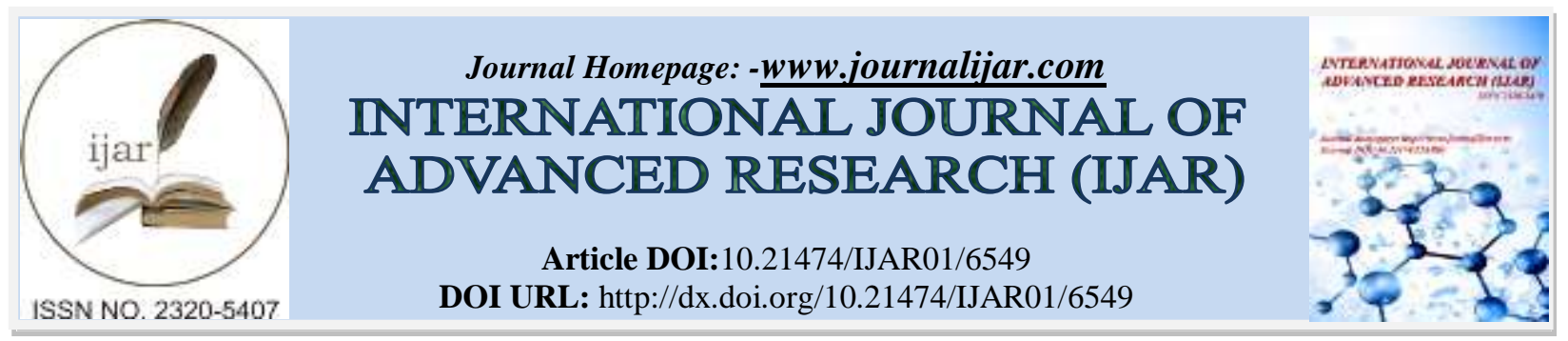

RESEARCH ARTICLE

\title{
ECHOCARDIOGRAPHIC VENTRICULAR DIASTOLIC FUNCTION FOR EVALUATION OF CHEMOTHERAPY-INDUCED CARDIOTOXICITY.
}

Essam M. Mahfouz, Wael M. Refaie, Manal A. Salah-Eldin and Wael A. Abdelmottaleb.

\section{Manuscript Info}

(.........................

Manuscript History

Received: 16 December 2017

Final Accepted: 18 January 2018

Published: February 2018

Keywords:-

Chemotherapy, Cardiotoxicity, Tissue Doppler Echocardiography.

\section{Abstract}

Background: The cardiotoxic effects of many chemotherapeutics are well reported. These effects vary from mild changes to serious complications. Tissue Doppler echocardiography was proposed as a useful diagnostic tool in patients affected by these changes.

Aim of work: The present study aimed to evaluate left \& right ventricles diastolic indices in patients on chemotheraputic treatment with doxorubicin and correlation of this parameters with some clinical, electrocardiographic and laboratory findings.

Patients and Methods: The present prospective study included 75 patients scheduled to receive doxorubicin therapy for different indications. They were subjected to careful history taking, thorough clinical examination, laboratory investigations and assessment of cardiac functions using ECG, PW Doppler and tissue Doppler before receiving chemotherapy and in two occasions after starting treatment (after the second cycle and the end of the treatment).

Results:In the current study, comparison between the studied patients and controls regarding the conventional echocardiographic parameters didn't show statistically significant differences. Prolonged QT is the most encountered ECG abnormality in the studied patients. Other changes included arrhythmia, low QRS, depressed ST, flattened and inverted T. Moreover, we found significant deterioration of diastolic dysfunction over the study period either using Doppler or tissue Doppler. In our study, patients with diastolic dysfunction were found to be older than patients without. However, the difference is not statistically significant. Regarding systolic dysfunction, the present study found systolic dysfunction in 4 patients $(5.3 \%)$.

Conclusions: Diastolic dysfunction is prevalent among patients under chemotherapy treatment. TDI provides a useful tool for early detection.

Copy Right, IJAR, 2018,. All rights reserved.

\section{Introduction:-}

Cardiotoxicity is a recognized chemotherapy-induced adverse event. As early as 1967, there were reports of heart failure in patients treated with doxorubicin (Tan et al., 1967). Since then the cytostatic antibiotics of the anthracycline class remain the most common cardiotoxic chemotherapeutic agents. There are, however, a number of other chemotherapeutic agents that cause cardiotoxicity which are not so well recognized by many clinicians. 
Cardiac events associated with chemotherapy vary from mild transient blood pressure and/or electrocardiographic (ECG) changes to more serious arrhythmias, myocarditis, pericarditis, myocardial infarction and cardiomyopathy, which may end in left ventricular dysfunction (LVD) or congestive heart failure (CHF) (Gharib and Burnett, 2002).

Among the imaging techniques, Doppler echocardiography offers several advantages: it can assess LV systolic and diastolic dysfunction, heart valve disease, pericarditis and pericardial effusion, and carotid artery lesions. LV fractional shortening and LVEF are the most common indexes used for LV systolic function for cardiac function assessment in oncology. LVEF is, however, not a very sensitive parameter in detecting early alterations in myocardial function. It is now recognized that the Doppler-derived diastolic indexes represent an early sign of LV dysfunction in cancer patients, so that evaluation of mitral diastolic flow pattern, early peak flow velocity to atrial peak flow velocity (E/A) ratio, deceleration time of $\mathrm{E}$ wave and isovolumic relaxation time can be useful to detect diastolic changes of LV function before systolic dysfunction occurs. Pulsed tissue Doppler may be easily performed during a standard Doppler echocardiographic examination; it has been successfully applied in several clinical setting and appears reliable in providing quantitative information on myocardial diastolic relaxation and systolic performance (E' wave, A' wave and S wave velocity). Tissue Doppler of LV lateral mitral annulus has a recognized prognostic role and, in combination with PW Doppler of mitral inflow, provides accurate information about the degree of LV filling pressure(Bovelli et al., 2010).

\section{Aim of work:-}

The present study aims to evaluate left and right ventricles diastolic indices in patients on chemotheraputic treatment with doxorubicin.

\section{Subjects and Methods:-}

The study was conducted on inpatients and outpatients at Mansoura Oncology Center as a prospective case control comparative study. The study included 75 adult patients on chemotherapy and 50 cancer patients not taking chemotherapy. Both groups were matched for age and sex distribution. Patients were excluded from the study if they had ischemic heart disease, systemic hypertension, chronic kidney disease, severe liver disease, COPD, cardiomyopathy or arrhythmia.

All participants were subjected to careful history taking, full cardiac examination, ECG and laboratory investigations including CBC, ESR, liver and kidney functions. The performed imaging studies included plain chest X-ray, and echocardiographic using VIVID 7 GE Medical System or a Sequoia Accuson ultrasound system with TDI capabilities. Patients were followed up after the second cycle and at end of treatment.

Data obtained from the present study were computed using SPSS versions 17 under the platform of Microsoft Windows 7. Continuous data were expressed in the form of mean \pm SD while categorical data were expressed in the form of count and percent. Comparison of continuous data was performed utilizing student $t$ test, while categorical data were done using Chi-square test. Relation between variables were investigation by Pearson's correlation coefficient. $\mathrm{P}$ value less than 0.05 was considered statistically significant.

\section{Results:-}

The present study included 75 patients in addition to 50 healthy controls. Comparison between patients and controls regarding the demographic, laboratory and echocardiographic data is shown in table-1. No statistically significant differences were found between the studied groups regarding age, gender distribution, laboratory data, and two dimensional echocardiography, Doppler echocardiography and tissue Doppler echocardiography parameters.

In table-2, follow up of ECG and echocardiographic data of the studied patients is illustrated. It showed significantly higher frequency of some ECG changes (prolonged QT, depressed ST, and flattened T) at the second follow up station. Two dimensional echocardiography showed significant deterioration of $\mathrm{EF}$ at first and second follow up stations. In addition, there were significant deterioration of Doppler parameters (E and E/A ratio) at the mitral valve and $\mathrm{E}, \mathrm{A}$ and $\mathrm{E} / \mathrm{A}$ ratio at the tricuspid valve. Moreover, TDI showed significant deterioration of $\mathrm{E}, \mathrm{A}$ and $\mathrm{E} / \mathrm{A}$ ratio throughout the study period at the mitral and tricuspid annuli.

As revealed in table-3, diastolic dysfunction was diagnosed in $53(70.7 \%)$ and 57 patients at the first follow up station and in $60(80.0 \%)$ and $71(94.7 \%)$ patients at the second follow up station as detected by Doppler and tissue Doppler echocardiographic respectively. 
Comparison between patients with diastolic dysfunction and patients without regarding the demographic, clinical and laboratory data didn't reveal statistically significant differences (Table-4).

Table-1:-Comparison between patients controls regarding the basic data

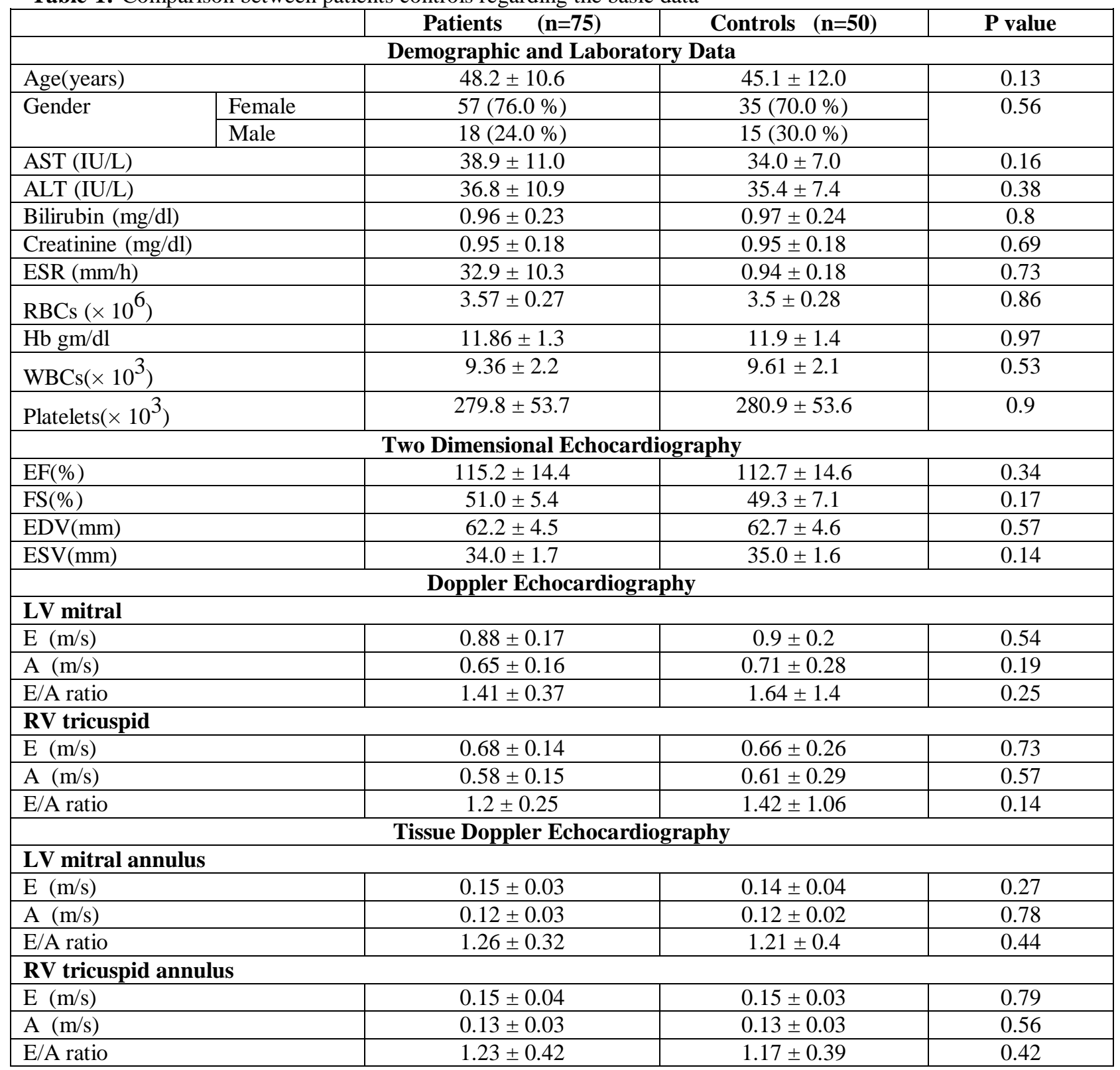

AST: Aspartate aminotransferase, ALT: Alanine aminotransferase, EDV: End diastolic volume, EF: Ejection fraction, ESR: Erythrocyte sedimentation rate, ESV: End systolic volume. FS: Fractional shortening.

Table-2:-ECG and Echocardiographic changes throughout the study

\begin{tabular}{|c|c|c|c|c|}
\hline 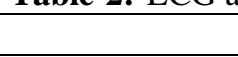 & Pretreatment & Follow up-1 & Follow up-2 & P value \\
\hline \multicolumn{5}{|c|}{ ECG } \\
\hline Arrhythmia & - & - & $1(1.33 \%)$ & 0.37 \\
\hline Low QRS & - & - & $2(2.67 \%)$ & 0.13 \\
\hline Prolonged QT & - & $7(9.33 \%)$ & $11(14.67 \%)$ & $0.004 *$ \\
\hline Depressed ST & - & - & $4(5.33 \%)$ & $0.017 *$ \\
\hline Flattened T & - & - & $5(6.67 \%)$ & $0.006^{*}$ \\
\hline Inverted T & - & - & $2(2.67 \%)$ & 0.13 \\
\hline
\end{tabular}




\begin{tabular}{|l|c|c|c|c|}
\hline \multicolumn{5}{|c|}{ Two Dimensional Echocardiography } \\
\hline EF\% & $62.2 \pm 14.5$ & $61.5 \pm 15.0$ & $60.1 \pm 15.3$ & $0.044^{*}$ \\
\hline FS\% & $34.0 \pm 1.7$ & $34.1 \pm 1.6$ & $34.3 \pm 1.6$ & 0.57 \\
\hline EDV & $115.2 \pm 14.4$ & $114.8 \pm 17.3$ & $112.1 \pm 15.7$ & 0.23 \\
\hline ESV & $51.0 \pm 5.4$ & $49.9 \pm 7.0$ & $50.0 \pm 7.3$ & 0.62 \\
\hline \multicolumn{5}{|c|}{ Doppler Echocardiography } \\
\hline LV mitral & $0.88 \pm 0.17$ & $0.79 \pm 0.17$ & $0.72 \pm 0.16$ & $0.0001^{*}$ \\
\hline E m/s & $0.65 \pm 0.16$ & $0.69 \pm 0.16$ & $0.71 \pm 0.16$ & 0.066 \\
\hline A m/s & $1.41 \pm 0.37$ & $1.23 \pm 0.44$ & $1.05 \pm 0.32$ & $0.0001^{*}$ \\
\hline E/A ratio & $0.68 \pm 0.14$ & $0.62 \pm 0.1$ & $0.61 \pm 0.09$ & $0.0001^{*}$ \\
\hline RV tricuspid & $0.58 \pm 0.15$ & $0.75 \pm .19$ & $0.77 \pm .19$ & $0.0001^{*}$ \\
\hline E m/s & $1.2 \pm 0.25$ & $0.9 \pm .36$ & $0.85 \pm 0.25$ & $0.0001^{*}$ \\
\hline A m/s & Tissue Doppler Echocardiography \\
\hline E/A ratio & \multicolumn{5}{|l|}{} \\
\hline \multicolumn{5}{|l|}{} \\
\hline LV mitral annulus & $0.15 \pm 0.03$ & $0.11 \pm 0.04$ & $0.10 \pm 0.03$ & $0.0001^{*}$ \\
\hline E & $0.12 \pm 0.03$ & $0.16 \pm 0.03$ & $0.20 \pm 0.04$ & $0.0001^{*}$ \\
\hline A & $1.26 \pm 0.32$ & $0.75 \pm 0.29$ & $0.54 \pm 0.22$ & $0.0001^{*}$ \\
\hline E/A ratio & $6.37 \pm 2.6$ & $7.8 \pm 4.1$ & $7.7 \pm 3.5$ & $0.017^{*}$ \\
\hline E/E' & $0.15 \pm 0.04$ & $0.12 \pm 0.03$ & $0.13 \pm 0.03$ & $0.0001^{*}$ \\
\hline RV tricuspid annulus & $0.13 \pm 0.03$ & $0.17 \pm 0.04$ & $0.17 \pm 0.04$ & $0.0001^{*}$ \\
\hline E & $1.23 \pm 0.42$ & $0.83 \pm 0.35$ & $0.76 \pm 0.3$ & $0.0001^{*}$ \\
\hline A &
\end{tabular}

Table-3:-Prevalence of diastolic dysfunction in the studied patients throughout the study period

\begin{tabular}{|c|c|c|c|c|c|c|}
\hline & \multicolumn{5}{|c|}{ Diastolic dysfunction } & \multirow{3}{*}{$P$ value } \\
\hline & \multicolumn{2}{|c|}{ Doppler Echocardiography } & \multirow[t]{2}{*}{$P$ value } & \multicolumn{2}{|c|}{$\begin{array}{c}\text { Tissue Doppler } \\
\text { Echocardiography }\end{array}$} & \\
\hline & + & - & & + & - & \\
\hline Pretreatment & - & $75(100.0 \%$ & $0.0001 *$ & - & - & $0.0001 *$ \\
\hline Follow up-1 & $53(70.7 \%)$ & $22(29.3 \%)$ & & $57(76.0 \%)$ & - & \\
\hline Follow up-2 & $60(80.0 \%)$ & $15(20.0 \%)$ & & $71(94.7 \%)$ & $4(5.3 \%)$ & \\
\hline
\end{tabular}

Table-4:- Comparison between patients with and without diastolic dysfunction as detected by TDI regarding the demographic, clinical and laboratory data.

\begin{tabular}{|c|c|c|c|c|}
\hline . & & $\begin{array}{c}\text { Diastolic } \\
\text { Dysfunction }(+) n=71\end{array}$ & $\begin{array}{c}\text { Diastolic Dysfunction } \\
(-) n=4\end{array}$ & P value \\
\hline \multicolumn{2}{|l|}{ Age } & $48.4 \pm 10.7$ & $44.2 \pm 6.5$ & 0.44 \\
\hline \multirow[t]{2}{*}{ Gender } & Female & 17 & 1 & \multirow[t]{2}{*}{0.96} \\
\hline & Male & 54 & 3 & \\
\hline \multirow[t]{3}{*}{ Malignancy } & Breast cancer & 54 & 3 & \multirow[t]{3}{*}{0.76} \\
\hline & NHL & 11 & 1 & \\
\hline & AML & 6 & - & \\
\hline \multicolumn{2}{|l|}{ AST } & $39.5 \pm 10.8$ & $29.5 \pm 11.7$ & 0.076 \\
\hline \multicolumn{2}{|l|}{ ALT } & $37.1 \pm 10.9$ & $32.2 \pm 11.2$ & 0.38 \\
\hline \multicolumn{2}{|l|}{ Bilirubin } & $0.96 \pm 0.23$ & $0.95 \pm 0.3$ & 0.89 \\
\hline \multicolumn{2}{|l|}{ Creatinine } & $0.95 \pm 0.18$ & $0.95 \pm 0.23$ & 0.97 \\
\hline \multicolumn{2}{|l|}{ ESR } & $32.8 \pm 10.3$ & $35.5 \pm 11.2$ & 0.61 \\
\hline \multicolumn{2}{|l|}{ RBCs } & $3.58 \pm 0.27$ & $3.45 \pm 0.37$ & 0.34 \\
\hline \multicolumn{2}{|l|}{ Hb } & $11.9 \pm 1.3$ & $11.1 \pm 1.08$ & 0.27 \\
\hline \multicolumn{2}{|l|}{ WBCs } & $9.41 \pm 2.23$ & $8.5 \pm 2.09$ & 0.42 \\
\hline
\end{tabular}




\begin{tabular}{|l|c|c|c|}
\hline Platelets & $278.0 \pm 53.6$ & $311.2 \pm 50.9$ & 0.23 \\
\hline
\end{tabular}

NHL: Non-Hodgkin lymphoma, AML: Acute myeloid leukemia.

\section{Discussion:-}

Cardiotoxicity is a recognized chemotherapy-induced adverse event. Since their discovery more than 50 years ago, anthracyclines (AC) have become the mainstay for treatment of many cancers. However, AC use is associated with a risk of heart failure proportionate to the cumulative exposure (Sawyer, 2013). In a meta analysis of 18 studies including a total of 49,017 cancer patients of whom 22,815 receiving AC, clinical and subclinical cardiotoxicity occurred in $6.3 \%$ and $17.9 \%$ of patients respectively (Lotrionte et al., 2013).

Cardiac events associated with chemotherapy vary from mild transient blood pressure and/or ECG changes to more serious arrhythmias, myocarditis, pericarditis, myocardial infarction, cardiomyopathy and CHF (Gharib and Burnett, 2002).

Cardiac status during chemotherapy has been monitored by many diagnostic modalities including electrocardiography radionuclide angiocardiography and endomyocardial biopsy (Villani et al., 2006). Among imaging techniques, Echo-Doppler offer several advantages. It can assess LV systolic and diastolic dysfunction, heart valve affection and pericardial diseases. Also, it's simple, widely available, relatively cheap and non-invasive technique. In addition, new modalities like tissue Doppler and stress imaging provide more accurate evaluation of cardiac systolic and diastolic functions (Bovelli et al.,2010)

The aim of the present study was to evaluate left and right ventricular diastolic functions indices in patients treated with doxorubicin and correlate these parameters with some clinical, ECG and laboratory data. Comparative analysis of demographic data and laboratory parameters between patients and control groups before therapy revealed no significant difference regarding the clinical, laboratory, electrocardiographic and echocardiographic data.

In our study, there was significantly higher frequency of some ECG changes (prolonged QT, depressed ST, and flattened T) at the second follow up station. These results are in accordance with Huang et al., (2010) who found increase in heart rate in $3.2 \%$, low voltage QRS in $4.8 \%$, prolonged QT in 12\%, ST depression in $7 \%$, T-wave changes in $16 \%$ of patients Also, QT prolongation after AC treatment was reported by the studies of Schwatz et al., (1987) and Shan et al., (1996).

Follow up analysis of LV systolic parameters in the patients group revealed a significant decrease in LVEF on follow up. Also, there was a significant increase in the prevalence of LV systolic dysfunction at the second follow up station to 5.3\% of patients. Our results are similar to those of Toggweiler et al.,(2012) who found systolic dysfunction in 8 out of 187 patients after doxorubicin treatment and Daoud et al.,2008 who found systolic dysfunction in $(4.8 \%)$ of patients.

Follow up of Doppler indices of left and right ventricles revealed highly significant decrease in mitral peak E-wave velocity and E/A ratio, a non-significant increase in a mitral A-wave velocity, highly significant decrease in tricuspid peak E-wave velocity and E/A ratio and highly significant increase in A-wave velocity. Diastolic dysfunction was diagnosed in $53(70.7 \%)$ and $57(76.0 \%)$ patients at the first follow up station and in $60(80.0 \%)$ and $71(94.7 \%)$ patients at the second follow up station as detected by Doppler and tissue Doppler echocardiography respectively.

These results are in agreement with Tassan-Mangina et al.,(2006) who found deterioration of LV diastolic dysfunction on follow up of 20 patients after doxorubicin therapy, Nagy et al., (2008)who found impairment of diastolic function in $97.5 \%$ of 40 women with breast cancer treated with doxorubicin and Daoud et al.,(2008) who found a highly significant deterioration of LV diastolic function indices in 42 patients with non-Hodgkin lymphoma treated with doxorubicin. The better performance of TDI in detection of diastolic dysfunction was also reported by Sherief et al., (2012) who showed that pulsed TD indices of diastolic dysfunction are affected earlier than conventional Doppler parameters after AC therapy.

In conclusion, the present study confirmed the value conventional pulsed Doppler and more precisely by pulsed TD in early detection of diastolic function after chemotherapy, before other clinical and laboratory variables and that diastolic dysfunction usually precedes the systolic function in this situation. 


\section{References:-}

1. Bovelli D, Plataniotis G, Roila F and On behalf of the ESMO Guidelines Working Group. Cardiotoxicity of chemotherapeutic agents and radiotherapy-related heart disease. Annals of Oncology (2010) 21 (suppl 5):v277v282.

2. Daoud EM, Salah El-Din MA, Azmy EE, Abd El-All IA, Saleh YM. Clinical Value of Serial cTnI and QTc Measurements and its Correlation with Doppler Echocardiography in the Assessment of Cardiotoxicity of Patients Treated for Aggressive Non Hodgkin Lymphoma. Egypt Heart J 200860 (3): 239-248.

3. Gharib MI, Burnett AK. Chemotherapy-induced cardiotoxicity: current practice and prospects of prophylaxis. Eur J Heart Fail. 2002 Jun;4(3):235-42.

4. Huang C, Zhang X, Ramil JM, Rikka S, Kim L, Lee Y, Gude NA, Thistlethwaite PA, Sussman MA, Gottlieb RA, Gustafsson AB. Juvenile exposure to anthracyclines impairs cardiac progenitor cell function and vascularization resulting in greater susceptibility to stress-induced myocardial injury in adult mice. Circulation. 2010 Feb 9;121(5):675-83.

5. Lotrionte M, Biondi-Zoccai G, Abbate A, Lanzetta G, D'Ascenzo F, Malavasi V, Peruzzi M, Frati G, Palazzoni G. Review and meta-analysis of incidence and clinical predictors of anthracycline cardiotoxicity. Am J Cardiol. 2013 Dec 15;112(12):1980-4.

6. Nagy AC, Cserép Z, Tolnay E, Nagykálnai T, Forster T. Early diagnosis of chemotherapy-induced cardiomyopathy: a prospective tissue Doppler imaging study. PatholOncol Res. 2008 Mar;14(1):69-77.

7. Schwartz RG, McKenzie WB, Alexander J, Sager P, D'Souza A, Manatunga A, Schwartz PE, Berger HJ, Setaro J, Surkin L, et al. Congestive heart failure and left ventricular dysfunction complicating doxorubicin therapy. Seven-year experience using serial radionuclide angiocardiography. Am J Med. 1987 Jun;82(6):1109-18.

8. Shan K, Lincoff AM, Young JB. Anthracycline-induced cardiotoxicity. Annals of Internal Medicine 1996;125:47-58.

9. Sherief LM, Kamal AG, Khalek EA, Kamal NM, Soliman AA, Esh AM. Biomarkers and early detection of late onset anthracycline-induced cardiotoxicity in children. Hematology. 2012 May;17(3):151-6.

10. Tan C, Tasaka H, Yu KP, Murphy ML, Karnofsky DA. Daunomycin, an antitumor antibiotic, in the treatment of neoplastic disease. Clinical evaluation with special reference to childhood leukemia. Cancer. 1967 Mar;20(3):333-53.

11. Tassan-Mangina S, Codorean D, Metivier M, Costa B, Himberlin C, Jouannaud C,Blaise AM, Elaerts J, Nazeyrollas P. Tissue Doppler imaging and conventionalechocardiography after anthracycline treatment in adults: early and latealterations of left ventricular function during a prospective study. Eur JEchocardiogr. 2006 Mar;7(2):141-6.

12. Toggweiler S, Odermatt Y, Brauchlin A, Zander T, Müller A, Zuber M, Winterhalder R, Erne P. The clinical value of echocardiography and acoustic cardiography to monitor patients undergoing anthracycline chemotherapy. ClinCardiol. 2013 Apr;36(4):201-6.

13. Villani F, Meazza R, Materazzo C. Non-invasive monitoring of cardiac hemodynamic parameters in doxorubicin-treated patients: comparison with echocardiography. Anticancer Res. 2006 Jan-Feb;26(1B):797801. 